\title{
Vibrational G peak splitting in laterally functionalized single wall carbon nanotubes: Theory and molecular dynamics simulations
}

\author{
Alan B. de Oliveira ${ }^{a}$, ${ }^{\text {, }}$ Hélio Chacham ${ }^{\text {b }}$, Jaqueline S. Soares ${ }^{a}$, Taíse M. Manhabosco a \\ Hélio F.V. de Resende ${ }^{a}$, Ronaldo J.C. Batista ${ }^{a}$ \\ a Departamento de Física, Universidade Federal de Ouro Preto, Ouro Preto, MG 35400-000, Brazil \\ ${ }^{\mathrm{b}}$ Departamento de Física, Universidade Federal de Minas Gerais, Belo Horizonte, MG 30123-970 Brazil
}

\section{A R T I C L E I N F O}

\section{Article history:}

Received 1 June 2015

Received in revised form

7 September 2015

Accepted 26 September 2015

Available online 1 October 2015

\begin{abstract}
A B S T R A C T
We present a theoretical study of the vibrational spectrum, in the $G$ band region, of laterally hydrogenated single wall carbon nanotubes through molecular dynamics simulations. We find that bilateral hydrogenation - which can be induced by hydrogenation under lateral strain - causes permanent oval deformations on the nanotubes and induces the splitting of vibrational states in the G-band region. We propose that such splitting can be used as a Raman fingerprint for detecting nanotubes that have been permanently modified due to bilateral hydrogenation. In particular, our results may help to clarify the recent findings of Araujo and collaborators [Nano Lett. 12, 4110 (2012)] which have found permanent modifications in the Raman $G$ peaks of locally compressed carbon nanotubes. We have also developed an analytical model for the proposed phenomenon that reproduces the splitting observed in the simulations.
\end{abstract}

() 2015 Elsevier Ltd. All rights reserved.

\section{Introduction}

Carbon nanotubes are considered to be the ideal form of fibers with superior mechanical properties compared to the best carbon fibers and are excellent load-bearing reinforcements in composites $[1,2]$. The mechanical, thermal stability and heat-transport properties of carbon nanotubes have been studied by researchers, given the strength of the carbon-carbon bond [3]. Due to their high Young's modulus, carbon nanotubes are exceptionally stiff [4-6]. These properties indicate that single wall carbon nanotubes (SWCNT) may be used in many different kinds of applications, as for example reinforcement of composite materials [7] and fabrication of electronic devices [8].

SWCNT properties are highly affected by its surrounding environment. In order to achieve the stage of full technological applications, it is necessary to correlate such properties with environmental factors [9-12], including external forces [9,13-23]. For example, the electronic and structural properties of carbon nanotubes deposited on substrate are not yet clarified. In fact, recent experiments have shown a strong interaction between

\footnotetext{
* Corresponding author.

E-mail address: deoliveira.alanbarros@gmail.com (A.B. de Oliveira).
}

carbon nanotubes and substrate inducing important deformation of the nanotube on top of substrate $[10,24,25]$.

The identification of the chirality $(n, m)$ of an individual singlewalled nanotube from Raman scattering can be significantly affected by the surrounding environment. There are different results when the SWCNT is freely suspended or when interacting with the substrate [26-29]. Differences also appear when SWCNTs are deformed by applied strain [30,31], if it is uniform throughout the length of the SWCNT or not, etc [17].

The G-band is known to greatly facilitate the study of environmental effects upon carbon nanotubes in general. Such a frequency can be used for diameter characterization, to distinguish between metallic and semiconducting SWCNTs, through strong differences in their Raman lineshapes [32,33], to probe the charge transfer arising from doping a SWCNT; and to study the selection rules in the various Raman scattering processes and scattering geometries to cite a few examples.

It has been recently shown by Araujo and collaborators [34] that the breaking of SWCNTs cylindrical symmetry leads to the splitting of vibrational modes associated with $\mathrm{C}-\mathrm{C}$ vibrations in the plane transversal to the tube axis ( $\mathrm{G}^{-}$peak). In such an experiment radial deformations on SWCNTs were induced by the tip of an atomic force microscope (AFM) simultaneously with the measurement of the Raman $G$ band. The observed deformation-induced splitting of 
the $\mathrm{G}^{-}$peak was not fully reversible, that is, in some experiments the SWCNT did not recover the non-split state (observed before the contact with the AFM tip), after the AFM tip was retracted. Such a result shows that something prevents the SWCNT to recover its cylindrical symmetry after being compressed by the AFM tip. A possible explanation for the observed permanent $\mathrm{G}^{-}$splitting is the covalent bonding between the most curved parts of the tube and chemical species of the environment during the compression process $[35,36]$. The tip induced radial deformation which severely increased the local curvature in some parts of the tube. It was shown that locally, highly curved regions of carbon nanotubes strongly affects local reactivity by increasing the $\mathrm{sp}^{3}$ character of the $\mathrm{sp}^{2} \mathrm{C}-\mathrm{C}$ bonds [37-39]. Such a lateral functionalization may prevent the tube to recover its original curvature and/or provide an asymmetrical distribution of mass, which could result in the observed splitting in vibrational modes.

In order to test the hypothesis described above, we employed molecular dynamics simulations to investigate the effects of lateral functionalization in vibrational modes of SWCNTs. Our calculations show that even if a small fraction of the tube atoms bind to hydrogen atoms, the $\mathrm{G}^{-}$peak splits into distinct vertical and horizontal contributions (which we will call $\mathrm{G}_{\mathrm{v}}^{-}$and $\mathrm{G}_{\mathrm{h}}^{-}$, respectively). We also found that the magnitude of the $G^{-}$splitting $\left(>20 \mathrm{~cm}^{-1}\right.$ for more than 10 adsorbed hydrogens as can be seen Fig. 4) is much larger than the experimental frequency resolution $\left(2 \mathrm{~cm}^{-1}\right)$ of modern Raman setups. Supporting our simulation results we developed a simple model which explains the origin of such splitting in the $\mathrm{G}^{-}$peak in terms of changes in the tube curvature. The present work can contribute to understand how molecules in the environment may change SWCNT properties.

This paper is organized as follows. In Section 2 we present the molecular dynamics simulation details. Section 3 contains the simulation results while the analytical model is shown in Section 4. Section 5 is reserved for final remarks.

\section{Simulation details}

We performed molecular dynamics simulations using the LAMMPS package [40]. The system considered consists of a $(10,0)$ single wall carbon nanotube composed by 520 carbon atoms with length of approximately $55 \AA$ along to the $z$-axis (the longitudinal axis). Thus $x$ - and $y$-axis are the transversal ones.

We consider a bi-lateral functionalization of the carbon nanotubes, where hydrogen atoms are chemically bound to opposite sides of the nanotubes (see Figs. 1 and 2). Only four rows of the tube (two on each side) are allowed to adsorb hydrogens. Each row have 26 carbon atoms, thus the maximum number of adsorbed hydrogens is 104. In this work, we considered cases in which up to 20 hydrogens are randomly adsorbed in the carbon nanotubes. Our simulations show that at temperature $T=300 \mathrm{~K}$ hydrogenated SWCNTs become unstable if more than 20 hydrogens are adsorbed, and the so called unzipping effect is observed. We notice that at $T=100 \mathrm{~K}$ the tubes become stable even when $104 \mathrm{H}$ atoms are adsorbed. This result is consistent with the experimental findings

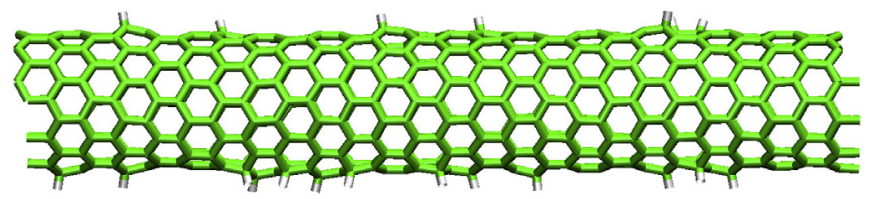

Fig. 1. Lateral view ( $x z$ - plane) of the hydrogenated SWCNT (twenty hydrogen atoms per unit cell) at $T=300 \mathrm{~K}$ and $P=1$ bar. (A color version of this figure can be viewed online.).

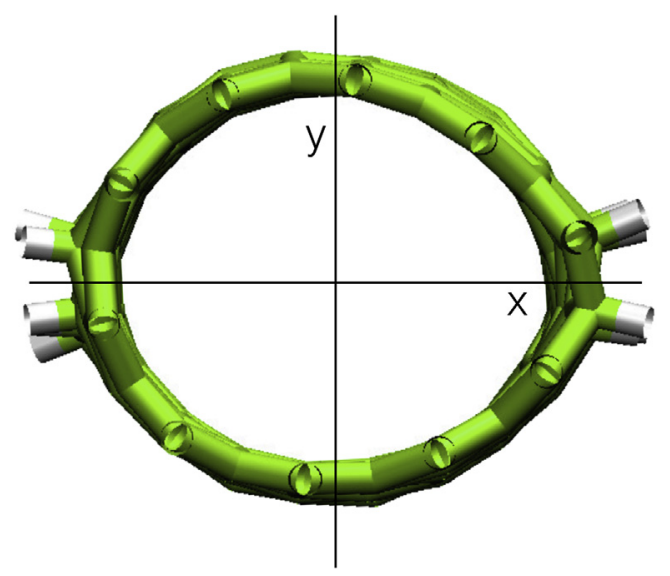

Fig. 2. Frontal view ( $x y$ - plane) of the hydrogenated SWCNT (twenty hydrogen atoms per unit cell) at $T=300 \mathrm{~K}$ and $P=1$ bar. (A color version of this figure can be viewed online.).

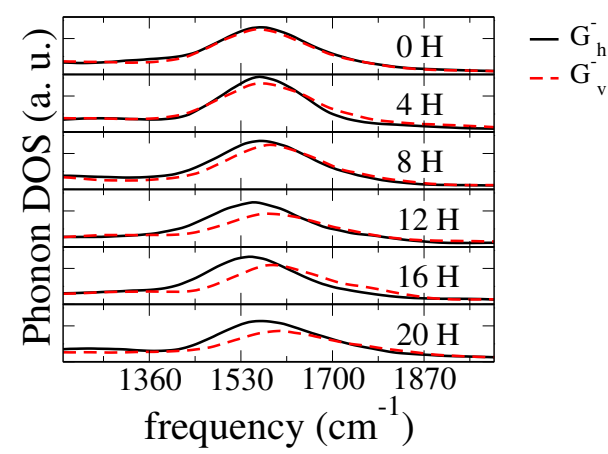

Fig. 3. Phonon DOS versus frequency for the hydrogenated SWCNT with 0 (perfect tube), $4,8,12,16$, and 20 hydrogens obtained through simulations for one of the five measurements. (A color version of this figure can be viewed online.).

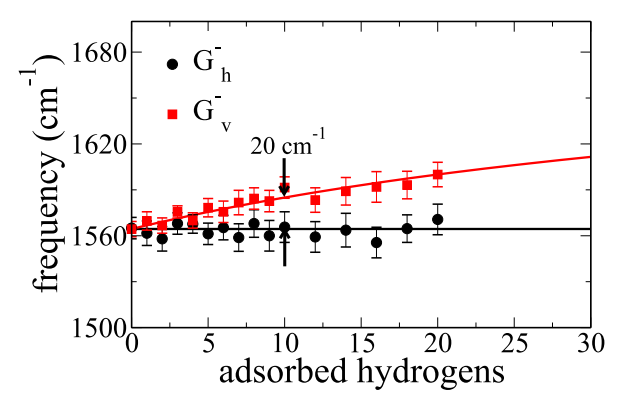

Fig. 4. Average positions of the maxima in the transverse modes of the G-band, $G_{h}^{-}$ (horizontal) and $\mathrm{G}_{\mathrm{v}}^{-}$(vertical), over five measurements for hydrogen adsorptions from 0 up to 20 . Error bars were obtained by calculating the standard deviations over five measurements. Symbols are data from simulations and lines are the results from our theoretical model, developed in Section 4. We see that at $10 \mathrm{H}$ the separation between $\mathrm{G}_{\mathrm{h}}^{-}$and $\mathrm{G}_{\mathrm{v}}^{-}$is about $20 \mathrm{~cm}^{-1}$ which is 10 times the resolution of modern Raman apparatuses. (A color version of this figure can be viewed online.).

which show that hydrogenated carbon nanotubes unzip at temperatures above certain critical value [41].

Periodic boundary conditions were considered in all directions with the simulation box, which has dimensions of $100 \AA$ in the $x$ and $y$-directions and $55.26 \AA$ along the $z$-axis. Those dimensions have shown to be suitable for simulating an infinite tube along its longitudinal axis whereas isolated laterally.

The timestep used was $\delta t=0.01 \mathrm{fs}$. Pressure and temperature 
were kept constant at 1 bar and $300 \mathrm{~K}$, respectively, by using the Nosé-Hoover thermostat [42] and barostat [43]. Finally, interactions between atoms $(\mathrm{C}-\mathrm{C}, \mathrm{C}-\mathrm{H}$, and $\mathrm{H}-\mathrm{H})$ were modeled classically with the ReaxFF potential [44].

Before the production stage, i.e., the stage in which the data are collected, there was an equilibration stage. In the equilibration stage the temperature was kept at $300 \mathrm{~K}$ during $10 \mathrm{ps}$. After that, followed the production stage in which the velocities of the carbon atoms were collected for each 500 steps (or 5 fs) along 525000 steps (or 5.25 ps). To calculate the phonon DOS, 1024 data points were used among those 1050 collected. The phonon DOS is calculated through the Fourier transform of the velocity autocorrelation function, between the velocity $\vec{v}$ in a time $t_{0}$ and a later time $t$,

$f(t)=\frac{1}{N} \sum_{i=1}^{N} \vec{v}_{i}\left(t_{0}\right) \cdot \vec{v}_{i}(t)$,

where the summation runs over the $\mathrm{N}$ carbon atoms. In order to improve the sample data, this procedure was performed five times, in which every one has a different, random hydrogen distribution for a certain degree of hydrogenation. The average of vertical and horizontal contributions to the G-band were calculated and the standard deviation was computed based on the referred five measurements.

\section{Simulation results}

Our results from simulations are summarized in Figs. 3 and 4. Fig. 3 shows the transversal (in the $x y$ plane) contributions to the phonon density of states (DOS) to the G-band against the frequency. $\mathrm{G}_{\mathrm{h}}^{-}$and $\mathrm{G}_{\mathrm{v}}^{-}$distinguish between the horizontal ( $x$-axis) and vertical $(y$-axis) modes in the transverse plane, respectively. The projection of the G-modes into $x$ and $y$ contributions is made by considering the respective velocity components along $x$ and $y$ in Eq. (1), i.e., $v_{x}$ and $v_{y}$. The $0 \mathrm{H}, 4 \mathrm{H}, \ldots, 20 \mathrm{H}$ refer to the number of hydrogen atoms adsorbed in the nanotubes $(0 \mathrm{H}$ meaning the tube without hydrogens).

The first aspect that draws attention in Fig. 3 is the agreement between simulations and experimental data available in literature regarding the position of the $\mathrm{G}^{-}$peak. Raman spectroscopy shows that such a peak is found in the frequency range from 1520 to $1630 \mathrm{~cm}^{-1}$ (roughly centered at the $1582 \mathrm{~cm}^{-1}$ ). The frequencies of the various $\mathrm{G}$-band peaks depend on the diameter of the tube and on whether the nanotube is metallic or semiconducting [9]. As we can see from Fig. 3, our results lie around $1550 \mathrm{~cm}^{-1}$, well within the cited experimental range. Simulations using the AIREBO potential, for example, present results for the G-band upshifted by approximately $200 \mathrm{~cm}^{-1}$, which is 100 times the resolution of modern Raman setups $[34,45,46]$. In this sense, the ReaxFF potential became the natural choice.

For the symmetric, pristine tube $(0 \mathrm{H}$-case) the modes in $x$ - and $y$-direction ( $G_{\mathrm{h}}^{-}$and $\mathrm{G}_{\mathrm{v}}^{-}$, respectively) are virtually indistinguishable, as expected. As the number of $\mathrm{H}$ atoms increase, the $\mathrm{G}_{\mathrm{h}}^{-}$and $\mathrm{G}_{\mathrm{v}}^{-}$modes start to split and we observe that the vertical mode shifts to higher frequencies. The $\mathrm{G}^{+}$mode appears to be insensitive to the addition of hydrogens (not shown), which is not surprising. Since the tube hydrogenation affects the symmetry in its transverse plane it is not expected that the $\mathrm{G}^{+}$mode would suffer major displacements.

Fig. 4 shows the positions of the maxima for the $G_{h}^{-}$and $G_{v}^{-}$ modes as shown in Fig. 3 against the degree of the tube hydrogenation. From this figure we clearly confirm what we visually detect from Fig. 3, that is, the separation of vertical and horizontal modes in the transverse plane of the tube. We see that for $10 \mathrm{H}$ adsorbed in the tube ( $\sim 2 \%$ of carbon atoms) the peak separation is about $20 \mathrm{~cm}^{-1}$ which, in principle, should not be difficult to detect by using state-of-the-art Raman setups. Such modern apparatus can resolve frequency shifts as low as $2 \mathrm{~cm}^{-1}$. The lines seen in Fig. 4 were obtained by fitting the Eq. (5) from our 8 mass/spring model developed in Section 4.

Our results are consistent with the experiments and the molecular dynamics simulations performed by P. T. Araujo and collaborators [34]. In such work a very similar G-band splitting was detected by compressing a serpentine (long, snake shaped SWCNT) with an AFM tip. In our work the symmetry breaking comes from $\mathrm{H}-\mathrm{C} \mathrm{sp}{ }^{3}$ bonds, which tend to be tetrahedral, in opposition to the planar $\mathrm{C}-\mathrm{C} \mathrm{sp}{ }^{2}$ bonds. This affects the curvature of the tube (see Fig. 2) leading to asymmetry in the transverse plane. In both cases, i.e., compressing the nanotube and by adsorbing H's in the tube, the effect become the tube deformation and consequently the splitting in the G-band. We argue in the Theoretical Model section that such a splitting comes from pure geometrical effects.

\section{Theoretical model}

In SWCNT systems, the G-band $\mathrm{G}^{-}$mode is an in plane stretching mode along the circumferential direction and the $\mathrm{G}^{+}$ mode is an in plane stretching mode along the tube axis [47-50]. Therefore, it is natural that the $\mathrm{G}^{-}$mode is very sensitive to changes in the SWCNT curvature while the $\mathrm{G}^{+}$is not. This curvature effect naturally appears in SWCNT with different diameters and the $\mathrm{G}^{-}$ mode frequency is softened with decreasing tube diameter, while the $\mathrm{G}^{+}$mode remains unaltered $[47,48,51-53]$.

Inspired by this curvature effect, we propose a simple model, consisting of eight identical masses $(\mathrm{m})$ and connected by eight identical springs $(\mathrm{k})$, to explain how transversal deformations change the $\mathrm{G}^{-}$mode behavior [see Fig. 5(a)]. The $(10,0)$ nanotube studied in this work has more than 8 atoms along the circumference. However, to obtain analytical solutions under the symmetry constraints of the problem, eight masses are largest possible system. Due to transversal deformations [see Fig. 5(b)], the tube circumference flattens leading to different vertical and horizontal normal modes. We will show that such a normal modes splitting effect can be explained by purely geometrical arguments.

For developing our mass-spring model we start by building the behavior of vectors $\mathbf{r}_{1}, \mathbf{r}_{2}$, and $\mathbf{r}_{3}$ under system deformation. It is possible to derive the normal modes of the 8 masses/spring system by investigating the motion of the three masses localized by the three cited vectors due symmetry arguments. We assume that $\mathbf{r}_{1}$ decreases and $\mathbf{r}_{3}$ increases by the same amount $\delta r$ due to radial deformation. For $r_{2}$, we assume that its horizontal component increases by $c_{x}^{\prime} \delta r$ while its vertical one decreases by $c^{\prime}{ }_{y} \delta r$ when the system is deformed. Considering that the length of $\mathbf{r}_{1}, \mathbf{r}_{2}$, and $\mathbf{r}_{3}$ is $r_{0}$ in the symmetrical case and by simple inspection of Fig. 6, we conclude that

$$
\begin{aligned}
& r_{1}=r_{0}-\delta r \\
& r_{3}=r_{0}+\delta r \\
& X=\frac{r_{0}+c_{x} \delta r}{\sqrt{2}} \\
& Y=\frac{r_{0}-c_{y} \delta r}{\sqrt{2}},
\end{aligned}
$$

where $X$ and $Y$ are the components of $\mathbf{r}_{2}$. Here, $c^{\prime}{ }_{x}=c_{X} / \sqrt{2}$ and $c^{\prime} y=c_{y} / \sqrt{2}$. $c_{x}$ and $c_{y}$ are parameters related to the tube's response to external agents acting on its morphology. For example, $c_{x}=c_{y}=0$ means that $X$ and $Y$ remain unchanged $\left(X=Y=r_{0} / \sqrt{2}\right.$ and $\left.\theta_{0}=\pi / 4\right)$ while $\alpha_{y}$ decreases and $\alpha_{x}$ increases under external 

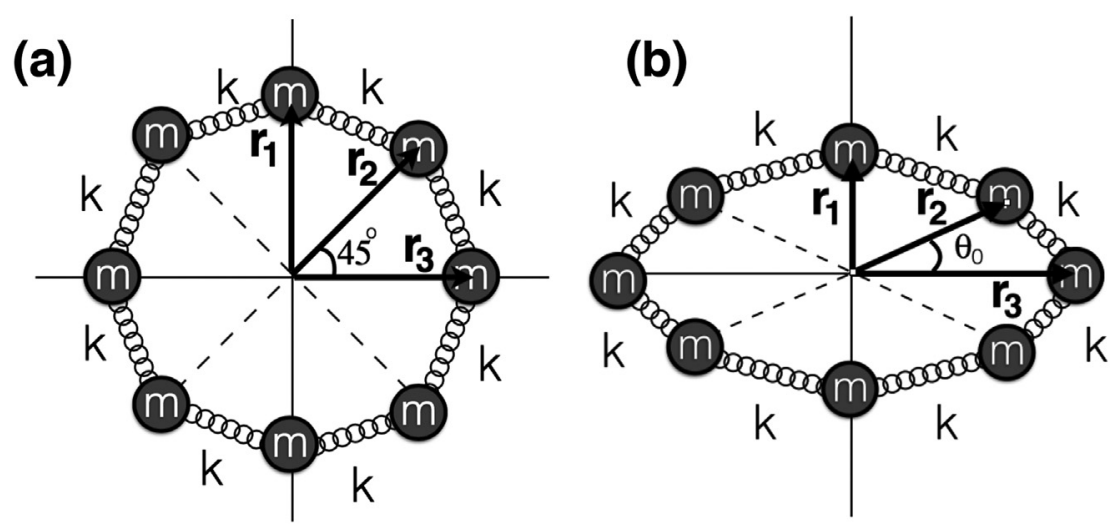

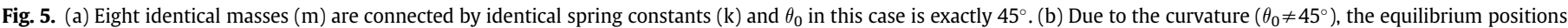
for the masses are changed and the normal modes in $x$ and $y$ directions become different.
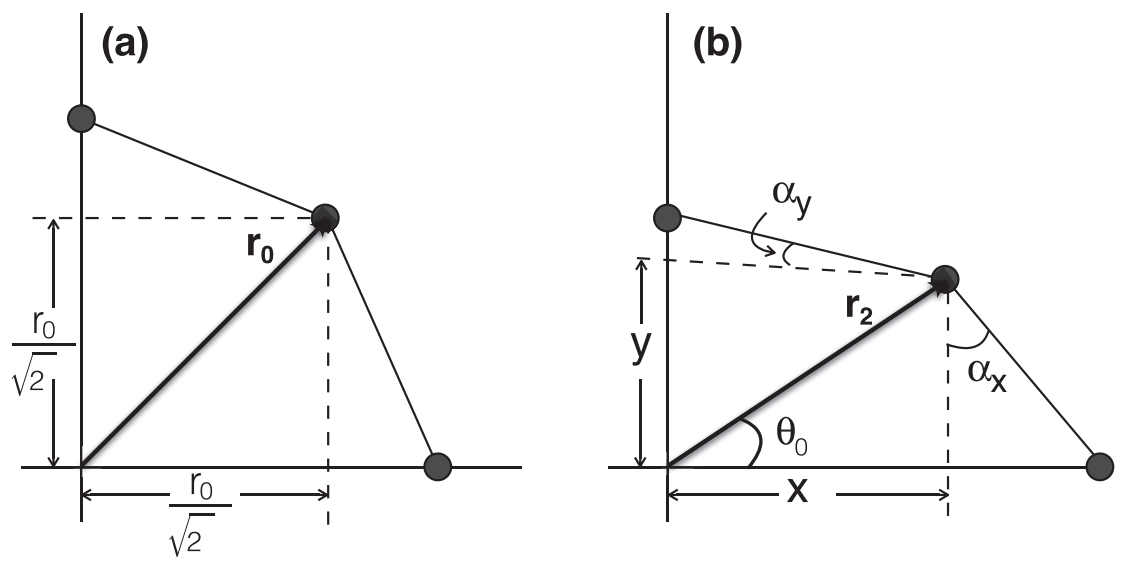

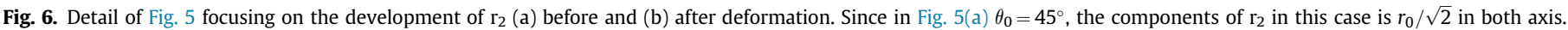

intervention (see Fig. 6). If $c_{X}=c_{y}=\sqrt{2}$, the deformation is entirely absorbed by $X$ and $Y$ with $\alpha_{x}$ and $\alpha_{y}$ keeping their initial values. Our choice for $\boldsymbol{r}_{1}$ and $\boldsymbol{r}_{3}$ given by Eq. (2) is based on the assumption that the $\mathrm{C}-\mathrm{C}$ bond is the hardest one in nature thus few susceptible to length variations. In this sense, we expect that the distance between masses are not sensible to system deformations and the perimeter of the ring must approximately coincides with the perimeter of the circle, $2 \pi r_{0}$, before deformation. We consider our 8 masses/spring system tend to assume an ellipsoidal shape when deformed, with $\boldsymbol{r}_{3}$ and $\boldsymbol{r}_{1}$ being the major and minor axis, respectively. With help of Eq. (2), its perimeter $p$ can be written as

$p=2 \pi r_{0}\left(1+\frac{1}{4} \gamma^{2}+\frac{1}{64} \gamma^{4}+\ldots\right)$

where we have defined the dimensionless quantity $\gamma \equiv \delta r / r_{0}$, which measures the deformation of the vector positions in relation to its original length. Note that $0 \leq \gamma<1$, where $\gamma=0$ refer to the symmetric case, whereas $\gamma \rightarrow 1$ means a tendency to the system to assume a bilayer conformation (highly deformed). For maintaining the same perimeter as the ring before deformation, we must neglect terms of degree 2 and higher in $\gamma$. We consider the maximum value for $\gamma=1 / 2$ at $20 \mathrm{H}$ (this is discussed later in the text) thus we estimate the maximum error due to this approximation of the order of 1/16 in comparison to the unity, which is not an absurd.

Within SWCNT symmetry, anti-symmetric modes belong to the
$E_{1}$ and $E_{2}$ irreducible representations and are not expected to show significant intensity in those spectra in Fig. 3, which are dominated by the totally symmetric $A_{1}$ modes [51]. The $G^{-}$modes observed in this work are symmetric so that it must be understood how symmetric modes are affected by transversal deformations. In this sense, we focused on the symmetric modes due to small dislocations from the equilibrium as shown in Fig. 7. The variations from the equilibrium of the vector positions $\mathbf{r}_{1}, \mathbf{r}_{2}$, and $\mathbf{r}_{3}$ are given by

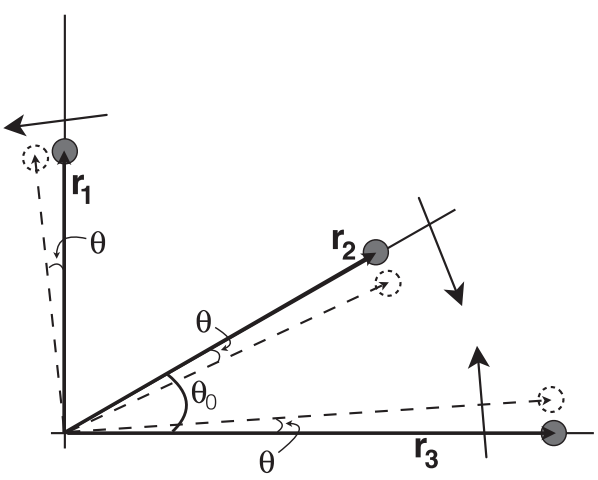

Fig. 7. Small angular displacement $\theta$ from equilibrium for the masses considered in our model. Exploring the symmetries of the system we only need to show the movement of masses localized by vectors $\mathbf{r}_{1}, \mathbf{r}_{2}$, and $\mathbf{r}_{3}$. Dashed lines and circles refer to the final position of the masses while the continuous ones to their initial positions. The arrows indicate dislocations in a motion restricted to angular displacements. 
$\begin{aligned} \Delta \mathbf{r}_{1} & =-r_{1} \widehat{\theta i} \\ \Delta \mathbf{r}_{2} & =r_{2} \theta\left(\sin \theta_{0} \widehat{i}-\cos \theta_{0} \widehat{j}\right) \\ \Delta \mathbf{r}_{3} & =r_{3} \theta \widehat{j}\end{aligned}$

where we have considered $\sin \theta \approx \theta$ and $\cos \theta \approx 1 . \widehat{i}$ and $\widehat{j}$ are the unit vectors pointing towards the positive direction of $x$ and $y$ axis, respectively. With help of Eq. (3), the displacement of the vector $\mathbf{r}_{2}$ relative $\mathbf{r}_{1}$ and $\mathbf{r}_{3}$ is given by

$\Delta \mathbf{R}=2 \Delta \mathbf{r}_{2}-\Delta \mathbf{r}_{1}-\Delta \mathbf{r}_{3}=\left(2 r_{2} \sin \theta_{0}+r_{1}\right) \hat{\theta i}-\left(2 r_{2} \cos \theta_{0}+r_{3}\right) \hat{j}$.

The equation of motion of the mass localized by $\mathbf{r}_{2}$ is given by $m \ddot{\mathbf{r}_{2}}=-k \Delta \mathbf{R}$, which leads to the equations $\theta \cdot+\omega_{\mathrm{h}}^{2} \theta=0$ and $\theta \cdot{ }^{*}+\omega_{\mathrm{v}}^{2} \theta=0$, corresponding to movements in $\mathrm{x}$ and y axis, respectively. With little calculation and using the Eq. (2), we find that the frequencies $\omega_{\mathrm{h}}$ (horizontal) and $\omega_{\mathrm{v}}$ (vertical) are given by

$\omega_{\mathrm{h}}^{*}=\left(2+\frac{r_{1}}{r_{2} \sin \theta_{0}}\right)^{1 / 2}=\left(2+\sqrt{2} \frac{1-\gamma}{1-c_{y} \gamma}\right)^{1 / 2}$

$\omega_{\mathrm{v}}^{*}=\left(2+\frac{r_{3}}{r_{2} \cos \theta_{0}}\right)^{1 / 2}=\left(2+\sqrt{2} \frac{1+\gamma}{1+c_{x} \gamma}\right)^{1 / 2}$,

with $\omega_{\mathrm{h}}^{*} \equiv \omega_{\mathrm{h}} \sqrt{m / k}$ and $\omega_{\mathrm{v}}^{*} \equiv \omega_{\mathrm{v}} \sqrt{m / k}$. Here we have used $X=r_{2} \cos \theta_{0}$ and $Y=r_{2} \sin \theta_{0}$ [see Fig. 6(b)].

According to our model Eq. (5), the symmetric mode for the system under no deformation $(\gamma=0)$ oscillates with frequency $\omega_{\mathrm{v}} \sqrt{m / k}=\omega_{\mathrm{h}} \sqrt{m / k}=(2+\sqrt{2})^{1 / 2}=1.85$. For increasing the asymmetry of the system $(\gamma \neq 0)$, the horizontal and vertical frequencies become different and increasingly split as seen in $A$ the $G^{-}$ mode of the G-band studied here. Eq. (5) were used to fit the data obtained through simulations and the resulting curves can be seen in Fig. 4. For doing that we linearly mapped the deformation parameter $\gamma$ into the number of hydrogens adsorbed as $\gamma=0$ at $0 \mathrm{H}$, while $\gamma=1 / 2$ at $20 \mathrm{H}$. The parameters used in the fits were $c_{x}=0.7$, $c_{y}=1.0$, and $\sqrt{\mathrm{k} / \mathrm{m}}=846.7 \mathrm{~cm}^{-1}$. Despite the simplicity of the model, the fits through the data obtained by using the Eq. (5) are very reasonable which supports our idea of explaining the G-band transversal mode splitting into a pure geometrical, mechanical perspective. Once the physics behind such an splitting is well stablished more sophisticated phenomena regarding the interaction between nanotubes and its surrounding environment can be explored with accordingly more complex theoretical approaches.

\section{Conclusion}

In this work we have investigated single walled carbon nanotubes laterally decorated with hydrogens at temperature $300 \mathrm{~K}$ and pressure 1 bar. The number of adsorbed H's in the tubes vary from 0 (pristine tube) up to 20 . For doing that we used the molecular dynamics techniques as implementes in the LAMMPS package [40] along with the ReaxFF potential for $\mathrm{C}-\mathrm{C}, \mathrm{C}-\mathrm{H}$, and $\mathrm{H}-\mathrm{H}$ interactions [44]. Our results show that even at low degrees of hydrogenation (for example, 10 adsorbed hydrogens in a 520 carbon atoms SWCNT which results in a adsorption of only $2 \%$ ) the $\mathrm{G}^{-}$peak (transversal modes) split into different horizontal and vertical contributions. For zero hydrogenation such contributions are identical as expected. The separation of the $\mathrm{G}^{-}$horizontal and vertical modes have been found to be about $20 \mathrm{~cm}^{-1}$ at $10 \mathrm{H}$, which is expected to be detectable by Raman spectroscopy experiments. Next, we propose a simple mass-spring model, composed by eight masses connected by eight springs, which shows the origin of the
$\mathrm{G}^{-}$splitting as purely geometrical, coming from curvature effect. In this work such a curvature is due to the tetrahedral nature of the $\mathrm{C}-\mathrm{H} \mathrm{sp}^{3}$ bonds, while in Araujo and collaborators work [34] the deformation comes from compressing long nanotubes using an AFM tip. In both cases, the $\mathrm{G}^{-}$mode splitting was observed. In this sense, this work can help to understand how environmental factors, such as different atmospheres for instance, can change SWCNT properties.

\section{Acknowledgments}

The authors thank the Brazilian funding agencies CNPq, CAPES, FAPEMIG, and the project INCT de Nanomateriais de Carbono for financial support.

\section{References}

[1] V.P. Veedu, A. Cao, X. Li, K. Ma, C. Soldano, S. Kar, et al., Multifunctional composites using reinforced laminae with carbon-nanotube forests, Nat. Mater. 5 (2006) 457-462.

[2] R.H. Baughman, A.A. Zakhidov, W.A. Zakhidov, Carbon nanotubes-the route toward applications, Science 297 (2002) 787-792.

[3] T. Yamamoto, S. Watanabe, K. Watanabe, Universal features of quantized thermal conductance of carbon nanotubes, Phys. Rev. Lett. 92 (2004) 075502.

[4] M.M.J. Treacy, T.W. Ebbesen, J.M. Gibson, Exceptionally high Young's modulus observed for individual carbon nanotubes, Nature 381 (1996) 678-680.

[5] J.P. Lu, Elastic properties of carbon nanotubes and nanoropes, Phys. Rev. Lett 79 (1997) 1297.

[6] E. Hernandez, C. Goze, P. Bernier, A. Rubio, Elastic properties of $\mathrm{C}$ and $\mathrm{B}_{x} \mathrm{C}_{y} \mathrm{~N}_{z}$ composite nanotubes, Phys. Rev. Lett. 80 (1998) 4502.

[7] J.N. Coleman, U. Khan, W.J. Blau, Y.K. Guníko, Small but strong: a review of the mechanical properties of carbon nanotubepolymer composites, Carbon 44 (2006) 1624-1652.

[8] A.M. Fennimore, T.D. Yuzvinsky, W.Q. Han, M.S. Fuhrer, J. Cumings, A. Zettl Rotational actuators based on carbon nanotubes, Nature 424 (2003) 408-410.

[9] M. Souza, A. Jorio, C. Fantini, B.R.A. Neves, M.A. Pimenta, R. Saito, et al., Singleand double-resonance raman G-band processes in carbon nanotubes, Phys. Rev. B 69 (2004) 241403.

[10] J.S. Soares, A.P.M. Barboza, P.T. Araujo, N.M.B. Neto, D. Nakabayashi, N. Shadmi, et al., Modulating the electronic properties along carbon nanotubes via tubesubstrate interaction, Nano Lett. 10 (2010) 5043-5048.

[11] A.P.M. Barboza, S.S. Carara, R.J.C. Batista, H. Chacham, B.R.A. Neves, Controlling the electrical behavior of semiconducting carbon nanotubes via tube contact. Small 8 (2) (2012) 220-224.

[12] R.J.C. Batista, A.B. de Oliveira, S.S. Carara, H. Chacham, Controlling the electrical response of carbon nanotubes deposited on diamond through the application of electric fields, J. Phys. Chem. C 118 (37) (2014) 21599-21603.

[13] P. Zhang, P.E. Lammert, V.H. Crespi, Plastic deformations of carbon nanotubes, Phys. Rev. Lett. 81 (1998) 5346-5349.

[14] T.W. Tombler, C. Zhou, L. Alexseyev, J. Kong, H. Dai, L. Liu, C.S. Jayanthi, et al. Reversible electromechanical characteristics of carbon nanotubes under localprobe manipulation, Nature 405 (2000) 767

[15] M.J. Peters, L.E. McNeil, J. Ping Lu, D. Kahn, Structural phase transition in carbon nanotube bundles under pressure, Phys. Rev. B 61 (2000) 5939-5944.

[16] S. Reich, C. Thomsen, P. Ordejn, Elastic properties of carbon nanotubes under hydrostatic pressure, Phys. Rev. B 65 (2002) 153407.

[17] S.B. Cronin, A.K. Swan, M.S. Ünlü, B.B. Goldberg, M.S. Dresselhaus, M. Tinkham, Measuring the uniaxial strain of individual single-wall carbon nanotubes: resonance raman spectra of atomic-force-microscope modified single-wall nanotubes, Phys. Rev. Lett. 93 (2004) 167401.

[18] B. Gao, J. Lai, L. Xi, L. Zhang, Z. Liu, Chirality-dependent raman frequency variation of single-walled carbon nanotubes under uniaxial strain, J. Phys Chem. C 112 (2008) 20123.

[19] A.G. Souza Filho, N. Kobayashi, J. Jiang, A. Grüneis, R. Saito, S.B. Cronin, et al. Strain- induced interference effects on the resonance raman cross section of carbon nanotubes, Phys. Rev. Lett. 95 (2005) 217403.

[20] X. Yang, G. Wu, J. Dong, Structural transformations of double-wall carbon nanotubes bundle under hydrostatic pressure, Appl. Phys. Lett. 89 (2006) 113101.

[21] M. Yao, Z. Wang, B. Liu, Y. Zou, S. Yu, W. Lin, et al., Raman signature to identify the structural transition of single wall carbon nanotubes under high pressure, Phys. Rev. B 78 (2008) 205411.

[22] A.P.M. Barboza, A.P. Gomes, B.S. Archanjo, P.T. Araujo, A. Jorio, A.S. Ferlauto, et al., Deformation induced semiconductor-metal transition in single wall carbon nanotubes probed by electric force microscopy, Phys. Rev. Lett. 100 (2008) 256804.

[23] A.L. Aguiar, E.B. Barros, R.B. Capaz, A.G. Souza Filho, P.T.C. Freire, J. Mendes Filho, et al., Pressure-induced collapse in double-walled carbon nanotubes: chemical and mechanical screening effects, J. Phys. Chem. C 115 (2011) 5378

[24] J.S. Soares, A. Jorio, Study of carbon nanotube-substrate interaction, 
J. Nanotechnol. 2012 (2012) 1-10.

[25] M. Steiner, M. Freitag, J.C. Tsang, V. Perebeinos, A.A. Bol, A.V. Failla, et al., How does the substrate affect the Raman and excited state spectra of a carbon nanotube? Appl. Phys. A 96 (2009) 271-282.

[26] Y. Zhang, J.Z.H. Son, M.S. Dresselhaus, J. Kong, Z. Liu, Raman spectra variation of partially suspended individual single-walled carbon nanotubes, J. Phys. Chem. C 111 (2007) 1983-1987.

[27] J.S. Soares, E.B. Barros, N. Shadmi, E. Joselevich, A. Jorio, Raman study of nanotube-substrate interaction using single-wall carbon nanotubes grown on crystalline quartz, Phys. Status Solidi B 248 (2011) 2536-2539.

[28] J.S. Soares, L.G. Cançado, E.B. Barros, A. Jorio, The kataura plot for single wal carbon nanotubes on top of crystalline quartz, Phys. Status Solidi B 247 (2010) 2835-2837.

[29] Y. Zhang, J. Zhang, H. Son, J. Kong, Z. Liu, Substrate-induced Raman frequency variation for single-walled carbon nanotubes, J. Am. Chem. Soc. 127 (2005) $17156-17157$.

[30] S. Ogata, Y. Shibutani, Ideal tensile strength and band gap of single-walled carbon nanotubes, Phys. Rev. B 68 (2003) 165409.

[31] A.G. Souza Filho, N. Kobayashi, J. Jiang, A. Grüneis, R. Saito, S.B. Cronin, et al. Strain-induced interference effects on the resonance Raman cross section of carbon nanotubes, Phys. Rev. Lett. 95 (2005) 217403.

[32] A. Jorio, M.A. Pimenta, A.G.S. Filho, R. Saito, G. Dresselhaus, M.S. Dresselhaus, Characterizing carbon nanotube samples with resonance Raman scattering New J. Phys. 5 (2003) 139.

[33] M.S. Dresselhaus, G. Dresselhaus, A. Jorio, A.G.S. Filho, G.G. Samsonidze R. Saito, Science and applications of single nanotube Raman spectroscopy, J. Nanosci. Nanotechnol. 3 (2003) 19-37.

[34] P.T. Araujo, N.M.N. Barbosa, H. Chachan, S.S. Carara, J.S. Soares, A.D. Souza, et al., In situ atomic force microscopy tip-induced deformations and Raman spectroscopy characterization of single-wall carbon nanotubes, Nano Lett. 12 (2012) 4110

[35] D. Stojkovic, P.E. Lammert, V.H. Crespi, Electronic bisection of a single-wall carbon nanotube by controlled chemisorption, Phys. Rev. Lett. 99 (2007) 026802.

[36] S.S. Alexandre, M.S.C. Mazzoni, H. Chacham, Edge states and magnetism in carbon nanotubes with line defects, Phys. Rev. Lett. 100 (2008) 146801.

[37] D. Srivastava, D.W. Brenner, J.D. Schall, K.D. Ausman, M. Yu, R.S. Ruoff, Predictions of enhanced chemical reactivity at regions of local conformational strain on carbon nanotubes: Kinky chemistry, J. Phys. Chem. B 103 (1999) 4330.
[38] K. Mylvaganam, L.C. Zhang, Deformation-promoted reactivity of single-walled carbon nanotubes, Nanotechnology 17 (2006) 410.

[39] S. Park, D. Srivastava, K. Cho, Generalized chemical reactivity of curved surfaces: carbon nanotubes, Nano Lett. 3 (2003) 1273.

[40] S.J. Plimpton, Fast parallel algorithms for short-range molecular dynamics, J. Comp. Phys. 117 (1) (1995). http://lammps.sandia.gov/.

[41] A.V. Talyzin, S. Luzan, I.V. Anoshkin, A.G. Nasibulin, H. Jiang, E.I. Kauppinen, et al., Hydrogenation, purification, and unzipping of carbon nanotubes by reaction with molecular hydrogen: road to graphane nanoribbons, ACS Nano 5 (2011) 5132.

[42] W.G. Hoover, Canonical dynamics: equilibrium phase-space distributions, Phys. Rev. A 31 (1985) 1695.

[43] W.G. Hoover, Constant-pressure equations of motion, Phys. Rev. A 34 (1986) 2499.

[44] A.C.T. van Duin, S. Dasgupta, F. Lorant, W.A. Goddard III, ReaxFF: a reactive force field for hydrocarbons, J. Phys. Chem. A 105 (2001) 9396.

[45] Z.-Y. Ong, E. Pop, Molecular dynamics simulation of thermal boundary conductance between carbon nanotubes and $\mathrm{SiO}_{2}$, Phys. Rev. B 81 (2010) 155408.

[46] Z.-Y. Ong, E. Pop, J. Shiomi, Reduction of phonon lifetimes and thermal conductivity of a carbon nanotube on amorphous silica, Phys. Rev. B 84 (2011) 165418.

[47] M. Dresselhaus, G. Dresselhaus, R. Saito, A. Jorio, Raman spectroscopy of carbon nanotubes, Phys. Rep. 409 (2) (2005) 47-99.

[48] A. Jorio, G. Dresselhaus, M.S. Dresselhaus, Carbon Nanotubes: Advanced Topics in the Synthesis, Structure, Properties and Applications, first ed. Springer-Verlag, Berlin, 2008.

[49] P. Jarillo-Herrero, S. Sapmaz, C. Dekker, L.P. Kouwenhoven, H.S.J. van der Zant, Electron-hole symmetry in a semiconducting carbon nanotube quantum dot, Nature 429 (2004) 389-392.

[50] H. Qian, P.T. Araujo, C. Georgi, T. Gokus, N. Hartmann, A.A. Green, et al., Visualizing the local optical response of semiconducting carbon nanotubes to DNA-wrapping, Nano Lett. 8 (9) (2008) 2706-2711.

[51] A. Jorio, M.S. Dresselhaus, R. Saito, G. Dresselhaus, Raman Spectroscopy in Graphene Related Systems, first ed., Swiley-VCH, Weinheim, 2011.

[52] O. Dubay, G. Kresse, H. Kuzmany, Phonon softening in metallic nanotubes by a peierls-like mechanism, Phys. Rev. Lett. 88 (2002) 235506.

[53] A. Jorio, M.A. Pimenta, A.G.S. Filho, G.G. Samsonidze, A.K. Swan, M.S. Ünlü, et al., Resonance Raman spectra of carbon nanotubes by cross-polarized light, Phys. Rev. Lett. 90 (2003) 107403. 